\title{
Unidimensional IRT Calibration of Compensatory and Noncompensatory Multidimensional Items
}

Terry A. Ackerman

American College Testing Program

The characteristics of unidimensional ability estimates obtained from data generated using multidimensional compensatory models were compared with estimates from noncompensatory IRT models. Reckase, Carlson, Ackerman, and Spray (1986) reported that when a compensatory model is used and item difficulty is confounded with dimensionality, the composition of the unidimensional ability estimates differs for different points along the unidimensional ability $(\theta)$ scale. Eight datasets (four compensatory, four noncompensatory) were generated for four different levels of correlated two-dimensional $\theta$ s. In each dataset, difficulty was confounded with dimensionality and then calibrated using LOGIST and BILOG. The confounding of difficulty and dimensionality affected the BILOG calibration of response vectors using matched multidimensional item parameters more than it affected the LOGIST calibration. As the correlation between the generated two-dimensional $\theta \mathrm{s}$ increased, the response data became more unidimensional as shown in bivariate plots of the mean $\hat{\theta}_{1}$ as opposed to the mean of $\hat{\theta}_{2}$ for specified unidimensional quantiles. Index terms. BILOG, compensatory IRT models, IRT ability estimation, LOGIST, multidimensional item response theory, noncompensatory IRT models.

One of the underlying assumptions of unidimensional item response theory (IRT) models is that a person's ability can be estimated in a unidimensional latent space. However, some researchers and educators doubt that the response process to any one item requires only a single latent ability. Traub (1983), for example, suggested that many cognitive variables are brought to the testing task and that the number used varies from person to person.

APPLIED PSYCHOLOGICAL MEASUREMENT

Vol. 13, No. 2, June 1989, pp. 113-127

(C) Copyright 1989 Applied Psychological Measurement inc. 0146-6216/89/020113-15\$2.00
Likewise, the combination of latent abilities that individuals use to obtain a correct response may vary from item to item. Reservations about applying unidimensional IRT estimation to multidimensional response data have been expressed by Ansley and Forsyth (1985), Reckase, Carlson, Ackerman, and Spray (1986), and Yen (1984), among others.

Reckase (1985) calibrated item response data from a form of the American College Test (ACT), Assessment Mathematics Usage Test, using the twodimensional IRT estimation program MAXLOG (McKinley \& Reckase, 1983) and found that easy items in the beginning of the test measured primarily one dimension and the more difficult items at the end of the test measured mostly along a second dimension. Thus difficulty appeared to be confounded with dimensionality. Using an updated version of MAXLOG, called MIRTE (Carlson, 1987), these results have been verified for several forms of the math usage test.

Using a compensatory multidimensional IRT model, Reckase et al. (1986) demonstrated that when dimensionality and difficulty are confounded (i.e., easy items discriminate only on $\theta_{1}$, difficult items discriminate only on $\theta_{2}$ ), the unidimensional $\theta$ scale has a different meaning at different points on the scale. Specifically, for their two-dimensional dataset, upper deciles of the unidimensional $\theta$ continuum differed mainly on $\theta_{2}$ while the lower deciles differed mostly on $\theta_{1}$. Thus, Reckase et al. suggested that the univariate calibration of twodimensional response data can be explained by the interaction between multidimensional test information and the distribution of the two-dimensional abilities. Reckase et al. examined the condition in 
which $\theta$ estimates were uncorrelated. Such an approach may not be very realistic, however, because most cognitive abilities tend to be correlated.

Ansley and Forsyth (1985) examined the unidimensional estimates from two-dimensional data generated using a noncompensatory model (Sympson, 1978). They selected item parameters so that generated response data would match item difficulty parameters as taken from a "real" test. They examined situations in which $\theta \mathrm{s}$ were correlated $0.0, .3, .6, .9$, and .95 . Although dimensionality might have been confounded with difficulty, the issue was not addressed. Ansley and Forsyth found that the $\hat{a}$ values were "best considered" as averages of the true $a_{1}$ and $a_{2}$ values, that the $\hat{b}$ values were "overestimates of $b_{1}$," and that $\hat{\theta}$ s were "highly related" to the average of the true $\theta_{1}$ and $\theta_{2}$ values.

Way, Ansley, and Forsyth (1988) compared the effects of using a unidimensional IRT model to estimate two-dimensional data generated by both noncompensatory and compensatory multidimensional IRT models. Results for the noncompensatory datasets were similar to those of the Ansley and Forsyth (1985) study. For the generated compensatory datasets, the $\hat{a}$ values were "best considered as an estimate of the sum of $a_{1}$ and $a_{2} " ; \hat{b}$ values were close to the "average of the $b_{1}$ and $b_{2}$ values"; and $\hat{\theta}$ was "highly related to the average" of the true $\theta_{1}$ and $\theta_{2}$ parameters.

This paper extends previous work by introducing new methodology to match compensatory and noncompensatory items, using simulated data based on multidimensional IRT calibration of real data using the compensatory model, and providing a graphical perspective from which the two models can be compared. Three issues are examined:

1. Do systematic differences exist between unidimensional calibrations of compensatory and noncompensatory items when difficulty is confounded with dimensionality? That is, would the results of the Reckase et al. (1986) study hold for both models?

2. Do different levels of correlation between twodimensional abilities affect the confounding of difficulty and dimensionality under each model? It was hypothesized that as the correlation between $\theta_{1}$ and $\theta_{2}$ increased, the response data would become essentially unidimensional, thus reducing the effect of confounding difficulty and dimensionality.

3. Do systematic differences exist between unidimensional item parameters and $\theta$ estimates obtained from the item calibration programs LOGIST and BLLOG when these programs are applied to either compensatory or noncompensatory multidimensional items?

\section{Model Definitions}

A multivariate logistic model introduced by Reckase (1985) was used to specify compensatory items. This model defines the probability of a correct response as

$$
\begin{aligned}
& P\left(x_{i j}=1 \mid a_{i}, d_{i}, \theta_{j}\right) \\
& =\frac{1}{1+\exp \left(d_{i}-\sum_{k=1}^{n} a_{i k} \theta_{j k}\right)},
\end{aligned}
$$

where $x_{i j}$ is the response to item $i$ by person $j$,

$\theta_{j k}$ is the ability parameter for person $j$ on dimension $k$,

$a_{i k}$ is the discrimination parameter for item $i$ on dimension $k$, and

$d_{i}$ is the difficulty parameter for item $i$.

The probability of a correct response for the noncompensatory logistic model introduced by Sympson (1978) is

$$
\begin{aligned}
& \mathbb{P}\left(x_{i j}=1 \mid \mathfrak{a}_{i}, \mathbb{B}_{i}, \mathfrak{c}_{i}, \theta_{j}\right) \\
& =c_{i}+\frac{1-c_{i}}{\prod_{k=1}^{n}\left\{1+\exp \left[-1.7 a_{i k}\left(\theta_{j k}-b_{i k}\right)\right]\right\}},
\end{aligned}
$$

where $x_{i j}, \theta_{j k}$, and $a_{i k}$ are defined as above,

$b_{i k}$ is the difficulty parameter for item $i$ on dimension $k$, and

$c_{i}$ is the guessing parameter for item $i$.

\section{Method}

\section{Data Generation}

To test the effects of correlated $\theta$ dimensions, four levels of correlation were selected $\left(\rho_{\theta_{1} \theta_{2}}=\right.$ $0.0,3, .6$, and .9). Parameters for a set of 40 two- 
dimensional compensatory items were selected with difficulty and dimensionality confounded. Discrimination parameters ranged from $a_{1}=1.8, a_{2}=$ .2 to $a_{1}=.2, a_{2}=1.8$. Difficulty was confounded with dimensionality such that the difficulty parameters ranged from $d=-2.4$ (for $a_{1}=1.8, a_{2}=$ .2) to $d=2.4$ (for $a_{1}=.2, a_{2}=1.8$ ). Thus as the items became more difficult, they discriminated less along $\theta_{2}$ and more along $\theta_{1}$. This systematic shift in dimensionality with increasing difficulty was established to mimic the pattern found in real data by Reckase (1985). Although perhaps not very realistic, the guessing parameter was set to 0 because of concern over how much "noise" would be added to the multidimensional data with a nonzero guessing parameter.

Instead of the trial-and-error methodology used by Way et al. (1988) to match compensatory items to noncompensatory items, a new methodology was developed. For each compensatory item, a corresponding noncompensatory item (same probability of a correct response) was created using a leastsquares approach to minimize the quantity

1000

$\sum_{j=1}\left[\left(P_{\mathrm{C}} \mid \mathbb{A}_{j}, \mathrm{a}, d\right)-\left(P_{\mathrm{NC}} \mid \mathbb{A}_{j}, \mathrm{R}, \mathrm{B}\right)\right]^{2}$,

where $P_{C}$ is a given compensatory item's probability of correct response, and $P_{\mathrm{NC}}$ is the noncompensatory item's probability of correct response (which varies as a function of and $\mathrm{b}$, given ${ }_{j}$ ). The summation was taken over 1,000 simulated examinees sampled from a bivariate normal population with $\mu_{1}=\mu_{2}=0, \quad \sigma_{\theta_{1}}=\sigma_{\theta_{2}}=1$, and $\rho_{\theta_{1} \theta_{2}}$ fixed at selected values. In all, four noncompensatory item sets were created with $\rho_{\theta_{1} \theta_{2}}=0.0$, 3, .6, and .9. Different starting values were tried to ensure that the function did have unique local minima where $P_{C}$ and $p_{\theta_{1} \theta_{2}}$ were fixed.

The mean, standard deviation, and minimum and maximum values of the difficulty and discrimination parameters for the compensatory and noncompensatory item sets are presented in Table 1 . Because the difficulty parameters for the compensatory model were symmetrical (i.e., $d_{1}=-d_{40}, d_{2}=$ - $d_{39}$, etc.), the mean difficulty is 0 . In each correlational condition, the noncompensatory $b_{1}$ is greatest and positive for item 1 and decreases stead- ily as the item number increases. However, noncompensatory difficulties for Dimension 2 are negative for all items for all correlational conditions.

The $a_{1}$ parameters for each model are greatest for item 1 and decrease with item number. The $a_{1}$ parameters are greater for the noncompensatory model and decrease at a slower rate than their compensatory counterparts. The $a_{2}$ parameters for each model are lowest for the first item and increase with item number. The $a_{2}$ parameters are greater for the noncompensatory model and increase at a slower rate than their compensatory counterparts.

Eight response datasets were then produced. Using the compensatory item parameters, 1,000 response vectors were simulated for each of four correlational values $\left(\rho_{\theta_{102}}=0.0, .3, .6, .9\right)$ from a bivariate normal distribution. For each set of noncompensatory item parameters, 1,000 response vectors were generated using the same $\left(\theta_{1}, \theta_{2}\right)$ combinations that produced the compensatory response datasets.

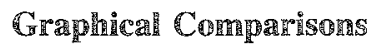

The generated compensatory item set is represented graphically in an item vector plot (Reckase, 1985), shown in Figure 1. In this plot the vector for each item represents the distance and direction from the origin to the point of maximum slope or discrimination. The $p=.5$ equiprobability line runs orthogonal to the tip of the item vector. Thus, the longer a vector extends into the third quadrant, the easier the item, and the longer a vector extends into the first quadrant, the more difficult the item.

To clarify how the probability of a correct response changes as a function of in each model, item response surfaces (IRSS) and corresponding contour plots for three pairs of matched items are presented in Figures 2 through 4. The IRS and its corresponding contour plot are shown for compensatory and noncompensatory items 1, 20, and 40 . Little difference exists among the IRSs for the matched items when the items discriminate only along $\theta_{2}$ (Figure 2) or only along $\theta_{1}$ (Figure 4). However, when both items discriminate equally along $\theta_{1}$ and $\theta_{2}$ (Figure 3), the noncompensatory equiprobability curves contrast sharply with the 
Table 1

Mean, Standard Deviation, Minimum and

Maximum for Generating Compensatory and Noncompensatory Item Parameters

\begin{tabular}{|c|c|c|c|c|}
\hline $\begin{array}{l}\text { Parameter } \\
\text { angi: Model }\end{array}$ & Mean & $\mathrm{SD}$ & Min & Max \\
\hline \multicolumn{5}{|c|}{ Compensatory } \\
\hline$a_{1}$ & 1.00 & .48 & .20 & 1.80 \\
\hline$a_{2}$ & 1.00 & .48 & .20 & 1.80 \\
\hline$d_{1}^{2}$ & .00 & 1.43 & -2.39 & 2.39 \\
\hline \multicolumn{5}{|c|}{ Noncompensatory: $r=0.0$} \\
\hline$a_{1}$ & 1.35 & .34 & .91 & 1.93 \\
\hline$a_{2}^{1}$ & 1.33 & .32 & .75 & 1.86 \\
\hline$b_{1}^{2}$ & -1.28 & 1.93 & -5.30 & 1.20 \\
\hline$b_{2}{ }^{1}$ & -1.29 & .56 & -3.22 & -.85 \\
\hline \multicolumn{5}{|c|}{ Noncompensatory: $x=.3$} \\
\hline$a_{1}$ & 1.47 & .31 & 1.01 & 1.96 \\
\hline$a_{2}^{1}$ & 1.44 & .33 & .81 & 1.93 \\
\hline$b_{1}^{2}$ & -1.08 & 1.67 & -4.59 & 1.15 \\
\hline$b_{2}^{1}$ & -1.11 & .42 & -2.58 & -.75 \\
\hline \multicolumn{5}{|c|}{ Nonicompensatory: $r=.6$} \\
\hline$a_{1}$ & 1.59 & .26 & 1.18 & 1.97 \\
\hline$a_{2}^{1}$ & 1.56 & .33 & .89 & 1.99 \\
\hline$b_{1}^{2}$ & -.90 & 1.44 & -3.86 & 1.13 \\
\hline$b_{2}^{1}$ & -.94 & .31 & -1.98 & -.63 \\
\hline \multicolumn{5}{|c|}{ Noncompensatory: $x=.9$} \\
\hline$a_{1}$ & 1.68 & .17 & 1.38 & 1.95 \\
\hline$a_{2}$ & 1.64 & .28 & 1.08 & 1.99 \\
\hline$b_{1}^{2}$ & -.73 & 1.26 & -3.28 & 1.12 \\
\hline$b_{2}^{1}$ & -.78 & .26 & -1.35 & -.49 \\
\hline
\end{tabular}

parallel equiprobability lines of the compensatory item.

In the compensatory model, the $\theta_{1} \theta_{2}$ combinations with an equiprobability of correct response form parallel lines. In contrast, for the noncompensatory model the $\theta_{1} \theta_{2}$ combinations with equiprobability of correct response are curvilinear. Also, unlike the compensatory model, in which the direction of the equiprobability lines is a function of the discrimination parameters, the direction of the equiprobability lines for the noncompensatory model is a function of the difficulty parameters. Thus, no constraints were placed on the noncompensatory difficulty parameters so that the equiprobability curves could be "shifted" to match compensatory items which measure mostly $\theta_{1}$ or $\theta_{2}$.
Another way to compare the two matched item sets is to examine the amount of test information each provides over the $\theta_{1} \theta_{2}$ ability plane. Multidimensional test information plots ("INFLINE" plots; Reckase, 1985) for one set of matched items $\left(\rho_{\theta 1 \theta_{2}}=0.0\right)$ are shown in Figures 5a (compensatory item set) and $5 \mathrm{~b}$ (noncompensatory item set). In each plot, the amount of test information at each of 49 selected points is illustrated by the length of the respective vectors for 10 different directions from $0^{\circ}$ to $90^{\circ}$ in $10^{\circ}$ increments. The maximum amounts of test information for the compensatory and noncompensatory item sets with $\rho_{\theta_{1} \theta_{2}}=0.0$ were 18.85 and 16.88 , respectively. For examinees with extremely high or extremely low $\theta$ on both dimensions, little information was provided for either 
item set. In general, for each level studied, more information was provided by the set of compensatory items relative to the matched noncompensatory set of items. However, for each item set, in the two-dimensional $\theta$ plane some isolated regions existed where this was not true.

\section{Ability and Item Parameter Estimation}

Each dataset was then calibrated using both LOGIST (Wingersky, Barton, \& Lord, 1982) and BILOG (Mislevy \& Bock, 1982). A two-parameter logistic IRT model was calibrated by both programs. The IRT calibration programs use different estimation procedures. LOGIST uses bounded joint maximum likelihood (JML) estimation. For the calibrations in this study, $\hat{a}$ was constrained to $\leqslant 2$ and the $c$ parameter was not estimated. For all BILOG computer runs the default method of scoring examinees was selected. The default method of $\theta$ estimation was expectation a posteriori using a normal 0,1 Bayesian prior. The default priors were also used in the item parameter calibration: a log-normal prior on the discrimination estimates and no prior on the difficulty estimates (Mislevy \& Stocking, 1989).

To estimate the LOGIST and BILOG orientation in the two-dimensional $\theta$ plane, the $\theta$ estimates from each calibration run were first rescaled to the compensatory $\theta$ estimates for the $\rho_{\theta_{1} \theta_{2}}=0.0$ case. The $\hat{\theta}$ values for each calibration run were rank-ordered and divided into 20 quantiles. The means of the $\theta_{1}$ and $\theta_{2}$ parameters for each $\hat{\theta}$ quantile were then calculated and plotted. These "centroid" plots were then examined for curvilinearity, which would suggest that the composite $\left(\theta_{1}, \theta_{2}\right)$ combination was not uniform across the univariate scale as predicted by the Reckase et al. (1986) study.

Results from the 16 sets of item calibrations were also evaluated by examining the correlations between $\hat{\theta}$ and $\theta_{1}\left(r_{\theta, \theta_{1}}\right)$, between $\hat{\theta}$ and $\theta_{2}\left(r_{\theta, \theta_{2}}\right)$, and

\section{Figure 1}

Vectors Representing the Distance and Direction From the Origin

to the Point of Maximum Discrimination for the 40 Generated Compensatory Items

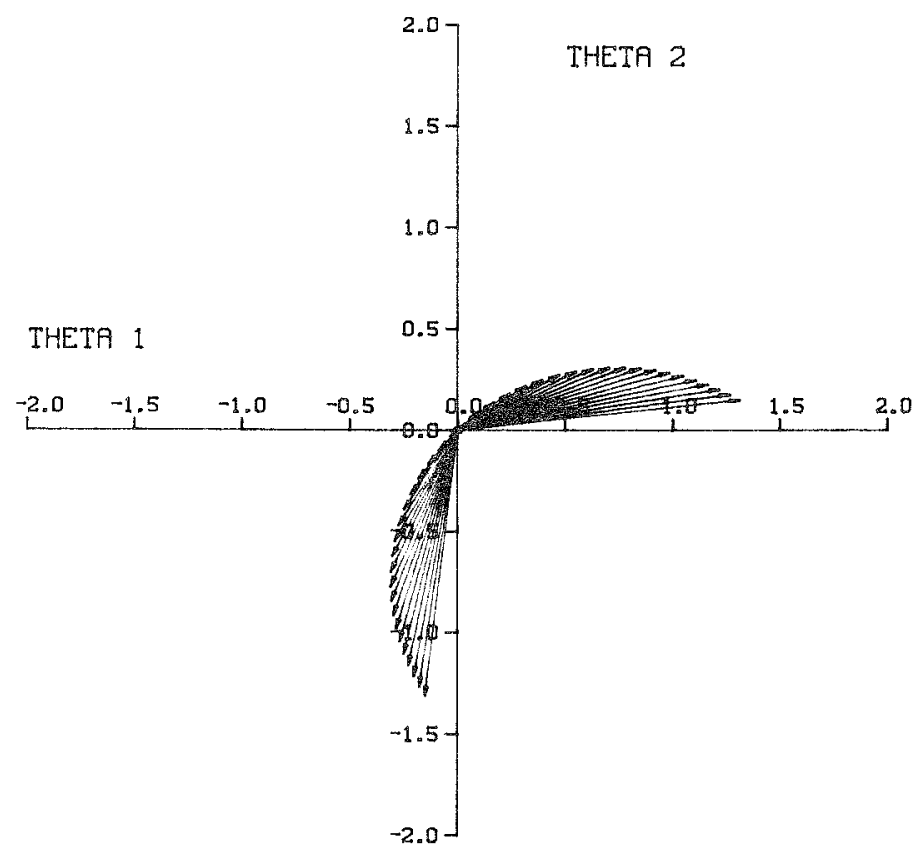


Tigure 2

The Item Response Surfaces and Contour Plots for Item 1

(a) Noncompensatory IRS

$\left(a_{1}=.91, a_{2}=1.85, b_{1}=-5.29, b_{2}=-1.35\right)$

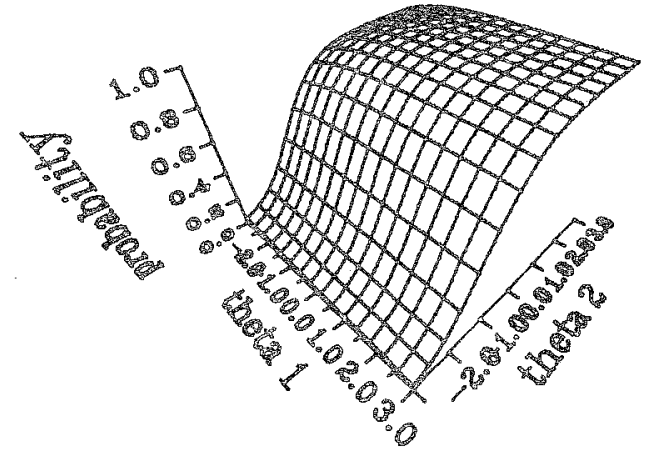

(c) Noncompensatory Contour Plot

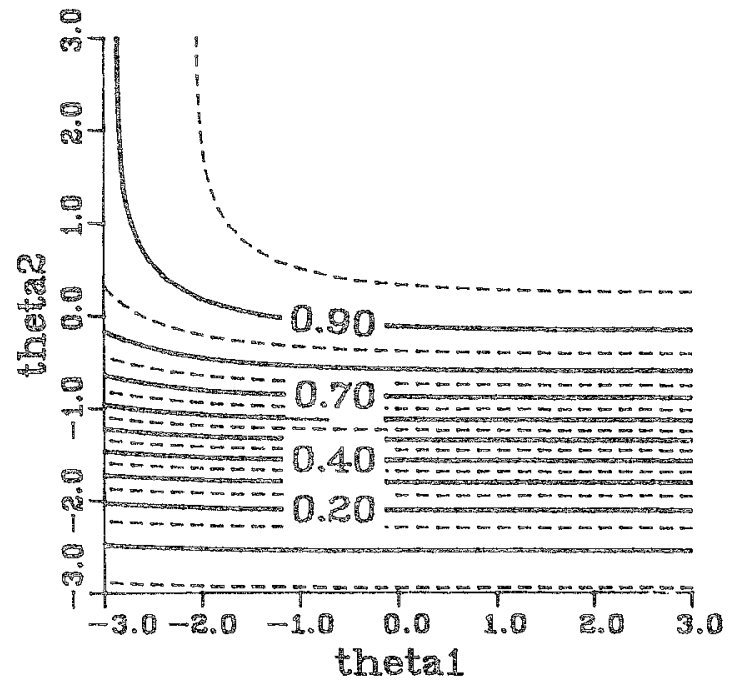

between $\hat{\theta}$ and the average of $\theta_{1}, \theta_{2}\left(r_{\theta, \theta_{\text {ave }}}\right)$. Mean absolute differences (MADS) were computed between $\hat{\theta}$ and $\theta_{1}\left[\left(\Sigma\left|\theta_{1}-\hat{\theta}\right|\right) / k\right]$, between $\hat{\theta}$ and $\theta_{2}$ $\left[\left(\Sigma\left|\theta_{2}-\hat{\theta}\right|\right) / k\right]$, and between $\hat{\theta}$ and the average of $\theta_{1}$ and $\theta_{2}\left[\left(\sum\left|\theta_{\text {ave }}-\hat{\theta}\right|\right) / k\right]$ for the $k$ simulated examinees.

Similar statistics were computed for the item discrimination and difficulty parameters and their unidimensional estimates to determine the effect of confounding difficulty with dimensionality for (b) Compensatory $\mathbb{R S}$

$\left(a_{1}=.20, a_{2}=1.80, d=2.39\right)$

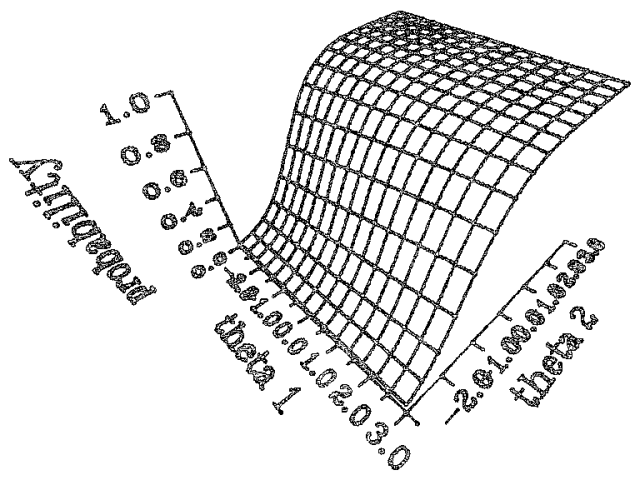

(d) Compensatory Contour Plot

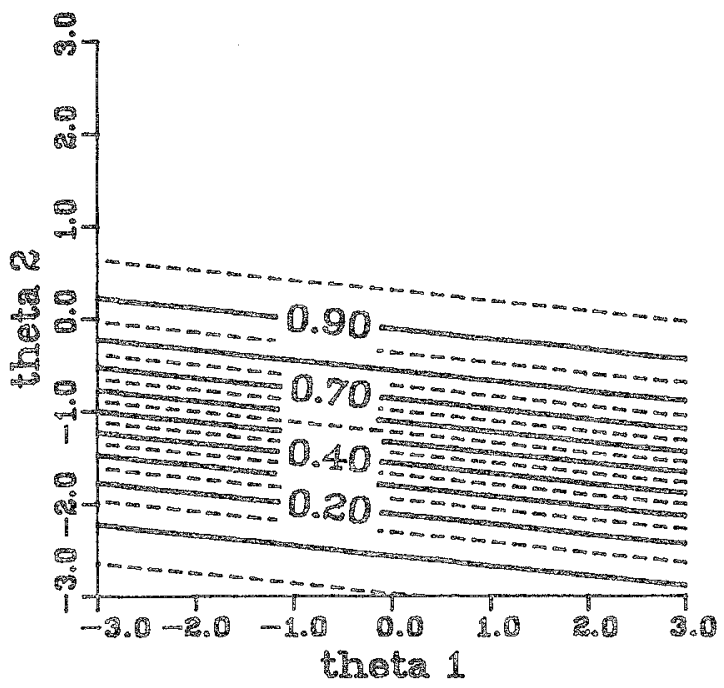

both the compensatory and noncompensatory item sets.

\section{Resullts}

Descriptive statistics for difficulty indices, itemtotal biserial correlations, KR-20 reliabilities, and the eigenvalues from a principal components anal$y$ sis of the tetrachoric correlation matrix were then obtained for each of the eight datasets, to validate 
Higure 3

The Item Response Surfaces and Contour Plots for Item 20

(a) Noncompensatory IRS

$\left(a_{1}=1.30, a_{2}=1.36, b_{1}=-.95, b_{2}=-.85\right)$

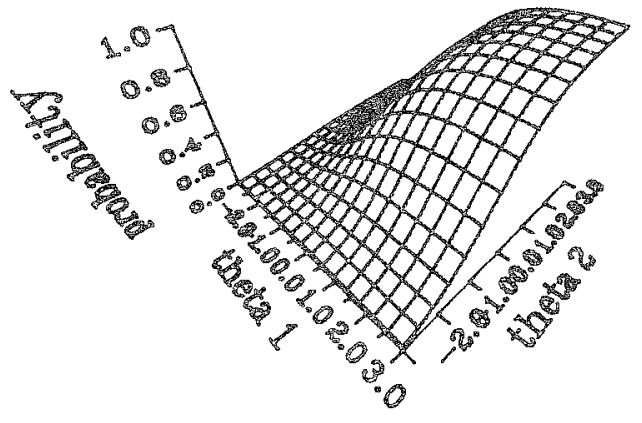

(c) Noncompensatory Contour Plot

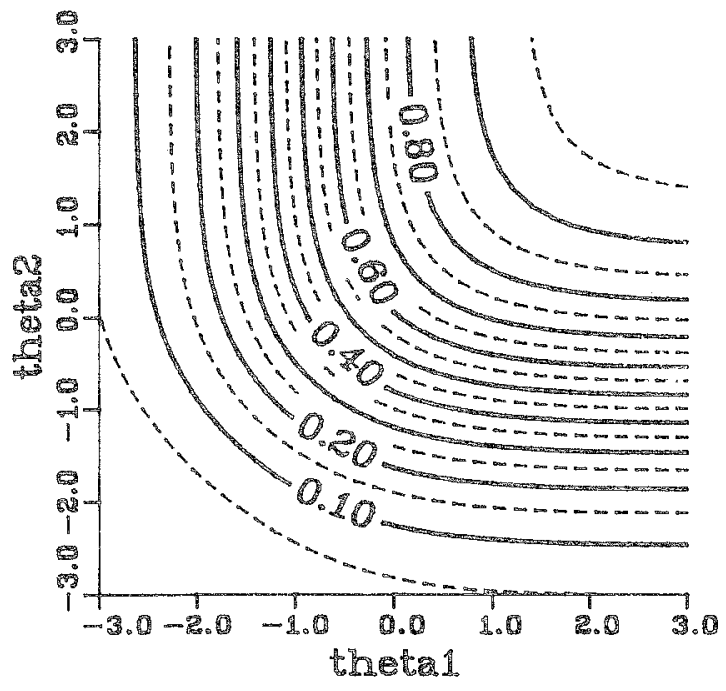

the similarities in item difficulty and to show the dimensionality of the data. These results are displayed in Table 2.

The eight item response sets have the same mean difficulty, with the range of $p$ values also similar. The mean biserials for compensatory and noncompensatory item sets appear more similar as the correlation between $\theta$ s increases. As the mean biserials increase, the KR-20 reliability coefficients also increase. Evidence of multidimensionality can be seen by forming a ratio of the first to the second (b) Compensatory IRS $\left(a_{1}=.98, a_{2}=1.02, d=.06\right)$

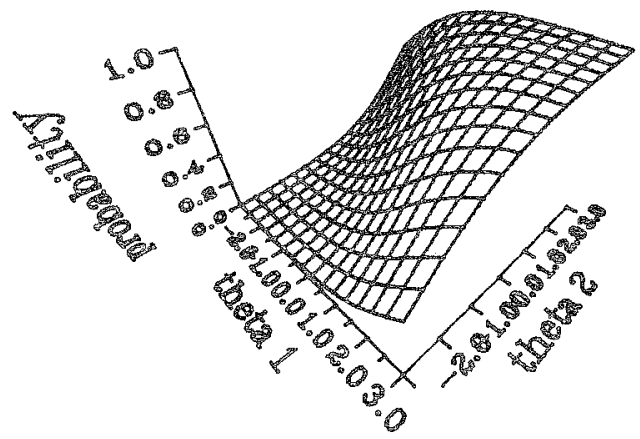

(d) Compensatory Contour Plot

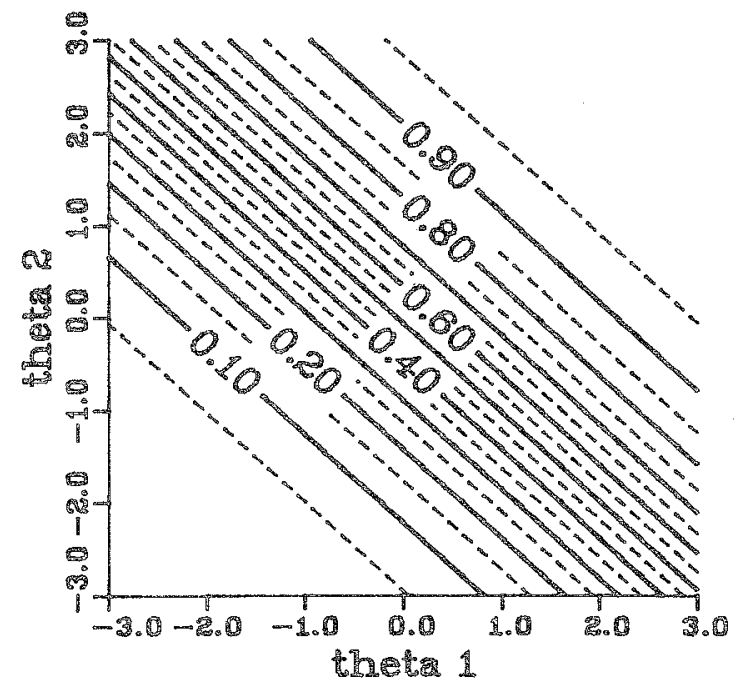

eigenvalue, $\lambda 1 / \lambda 2$ (Hambleton \& Murray, 1983). As the ccrrelation between the $\theta$ s increases, the ratio increases, which suggests a more dominant first principal component; at $\rho_{\theta_{1} \theta_{2}}=.9$ the data are almost unidimensional.

The LOGIST calibration program converged to a JML solution for each dataset. The maximum number of stages that LOGIST took to converge was 12 for the compensatory datasets $\left(\rho_{\theta_{1} \theta_{2}}=0.0\right)$ and 15 for the noncompensatory datasets $\left(\rho_{\theta_{1} \theta_{2}}=.3\right)$. The number of stages required for convergence was 
always greater for the noncompensatory dataset for each correlational level of the two-dimensional $\theta$ s. However, no pattern emerged between the correlational structure of the generating $\theta \mathrm{s}$ and the number of stages until convergence.

The centroid plots for the LOGIST calibration of the four compensatory and four noncompensatory datasets are shown in Figures $6 a$ and $6 b$, respectively. The BILOG counterparts are presented in Figures $7 a$ and $7 b$.

The LOGIST orientation is similar for each level of correlation and for each type of multidimen- sional model. The BILOG centroids are noticeably more variable. For the BILOG centroids, as $r_{\theta, \theta_{1}}$ approaches 0 , the plot of the centroids increases in curvature. Thus, BILOG is more sensitive to the confounding of difficulty and dimensionality. When the $\theta$ correlation is 9 , the centroids for both calibration programs are almost linear.

The correlations and MAD values for $\theta$ are shown in Table 3. Compared to the centroid plots, the data are much more alike for compensatory and noncompensatory datasets and for LOGIST estimates compared to BILOG's estimates. It is inter-

Tigure 4

The Item Response Surfaces and Contour Plots for Item 40

(a) Noncompensatory IRS

$\left(a_{1}=1.92, a_{2}=.75, b_{1}=1.19, b_{2}=-3.22\right)$

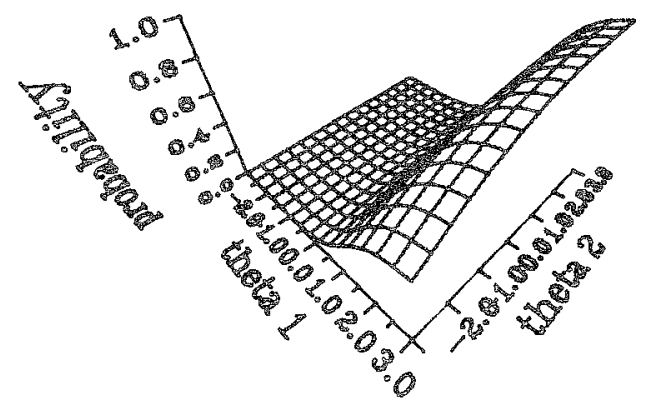

(c) Noncompensatory Contour Plot

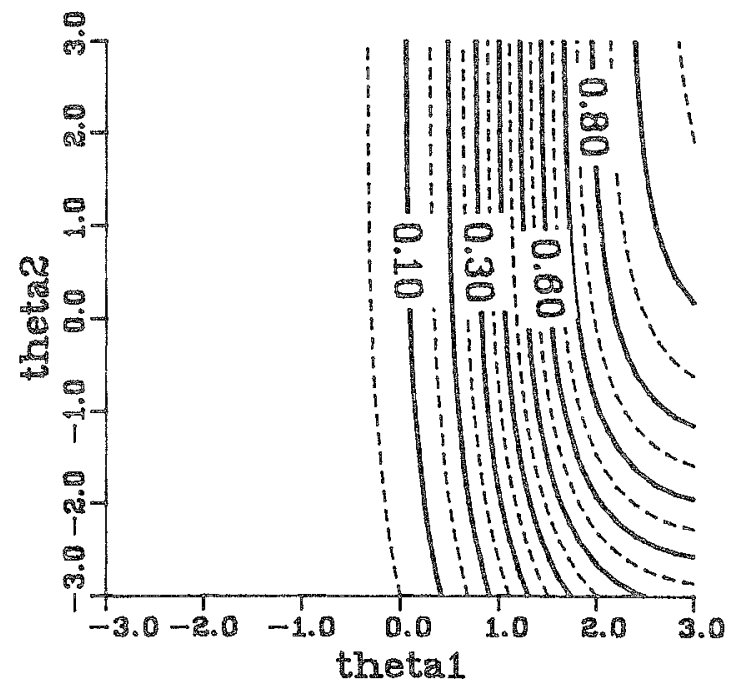

(b) Compensatory IRS

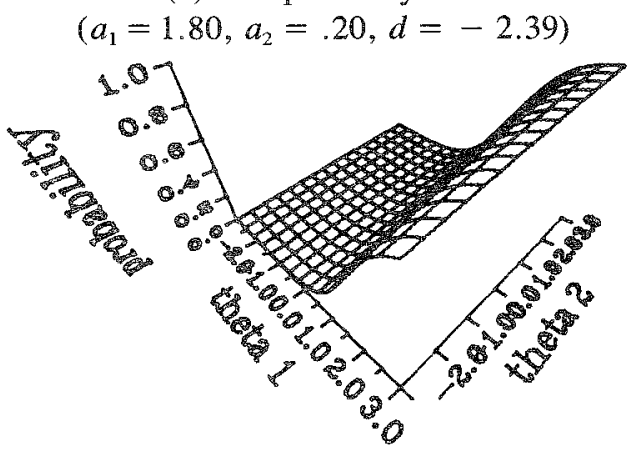

(d) Compensatory Contour Plot

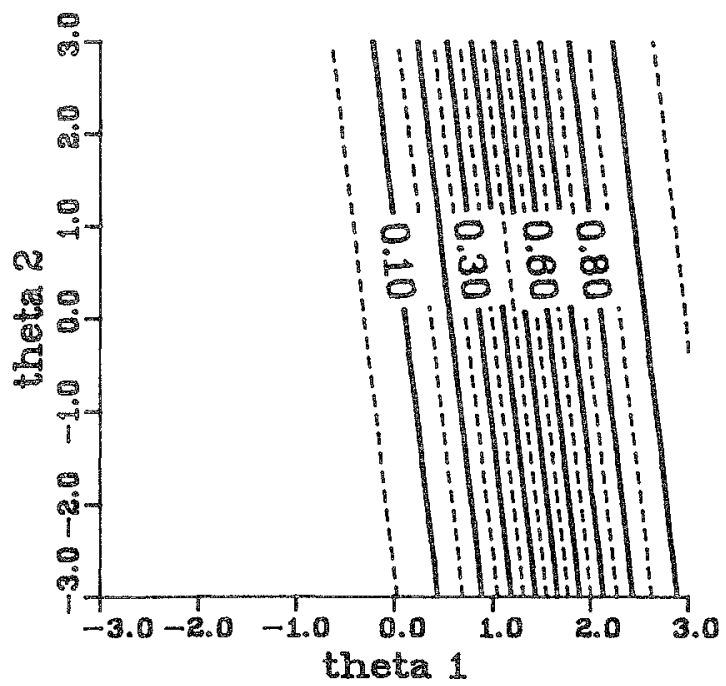


Figure 5

Test Information Vectors at Selected Points in the Ability Plane

(a) Compensatory

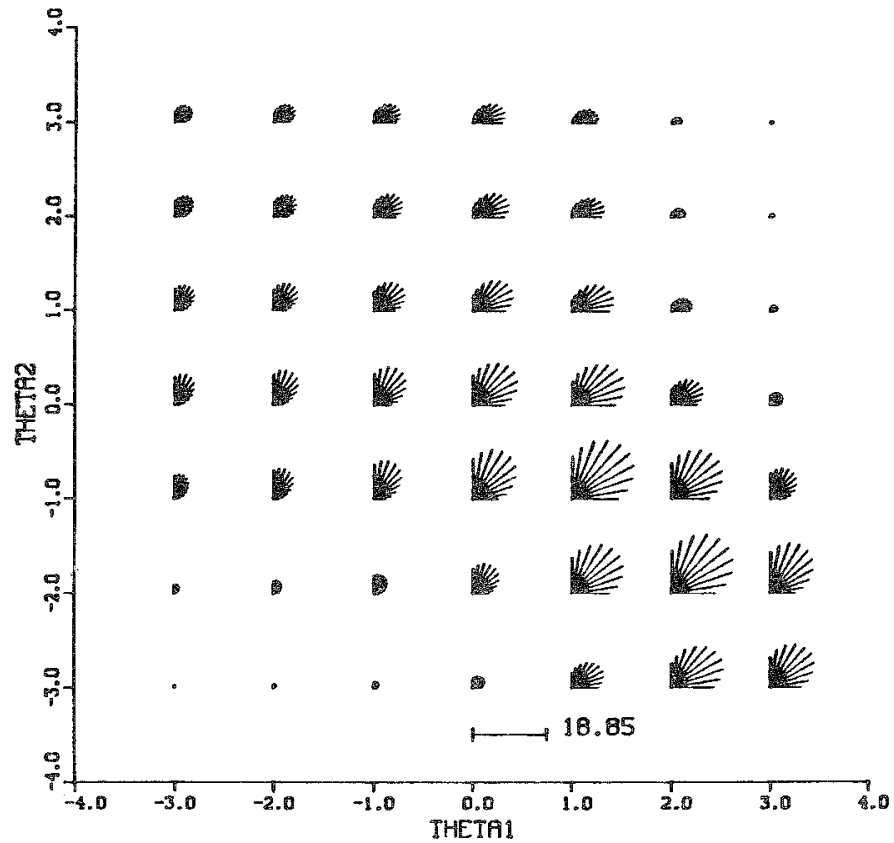

(b) Noncompensatory

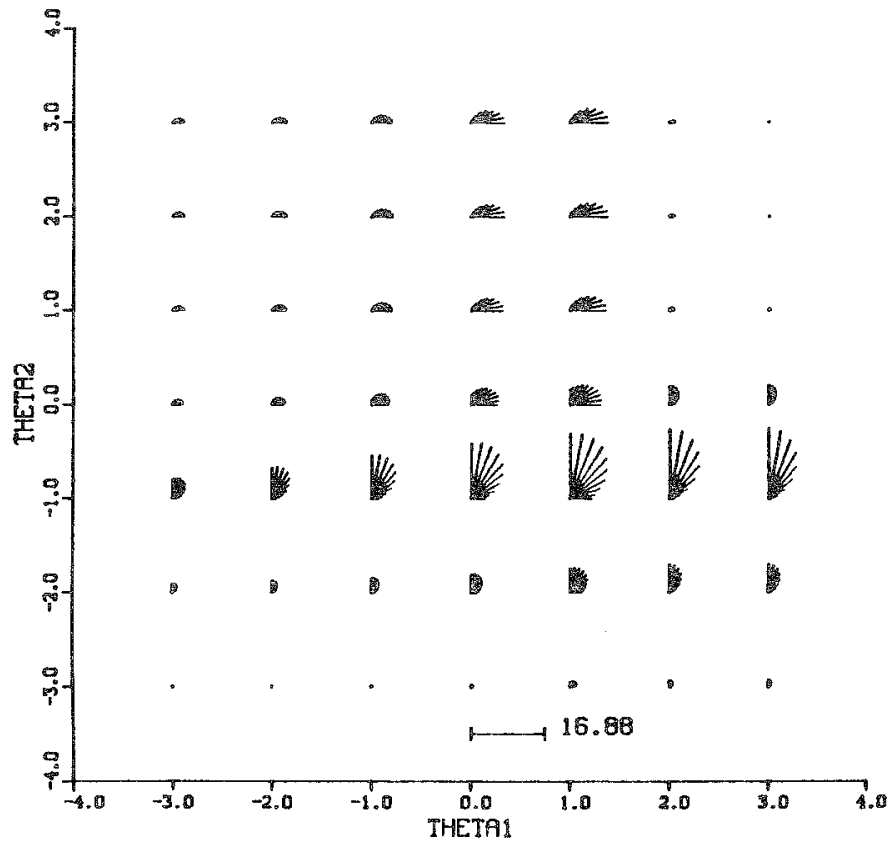


Table 2

Eigenvalues of the First and Second Principal Components of the Inter-Item Tetrachoric Correlation and Descriptive Statistics of the Multidimensional Datasets $(N=1,000, i=40)$

\begin{tabular}{|c|c|c|c|c|c|c|c|c|c|c|c|}
\hline \multirow{2}{*}{$\begin{array}{l}\text { Data Type } \\
\text { and } \rho\left(\theta_{1} \theta_{2}\right)\end{array}$} & \multicolumn{2}{|c|}{ Eigenvalues } & \multirow[b]{2}{*}{$K \mathbb{R}-20$} & \multicolumn{3}{|c|}{$\begin{array}{c}\text { Proportion } \\
\text { Correct }\end{array}$} & \multicolumn{3}{|c|}{$\begin{array}{l}\text { Biserial } \\
\text { Correlation } \\
\end{array}$} & \multicolumn{2}{|c|}{$\begin{array}{l}\text { Raw } \\
\text { Score }\end{array}$} \\
\hline & $\lambda_{1}$ & $\lambda_{2}$ & & Mean & Lo & $\overline{\mathrm{Hi}}$ & Mean & Lo & $\mathrm{Hi}$ & Mean & SD \\
\hline \multicolumn{12}{|c|}{ Compensatory } \\
\hline 0.0 & 9.24 & 2.94 & .91 & .50 & .16 & .85 & .64 & .50 & .71 & 20.15 & 8.64 \\
\hline .3 & 10.84 & 2.59 & .93 & .50 & .17 & .84 & .69 & .57 & .75 & 20.18 & 9.41 \\
\hline .6 & 12.17 & 2.27 & .94 & .50 & .18 & .84 & .73 & .59 & .79 & 20.15 & 10.04 \\
\hline .9 & 13.38 & 2.00 & .95 & .50 & .18 & .83 & .76 & .61 & .82 & 20.18 & 10.61 \\
\hline \multicolumn{12}{|c|}{ Noncompensatory } \\
\hline 0.0 & 7.22 & 3.17 & .88 & .50 & .16 & .84 & .56 & .47 & .64 & 20.03 & 7.64 \\
\hline .3 & 9.52 & 2.69 & .92 & .50 & .17 & 83 & .65 & .57 & .72 & 20.08 & 8.84 \\
\hline .6 & 11.64 & 2.25 & .94 & .50 & .17 & .84 & .71 & .65 & .76 & 20.13 & 9.82 \\
\hline .9 & 13.53 & 1.98 & .95 & .50 & .18 & .83 & .77 & .69 & .80 & 20.00 & 10.67 \\
\hline
\end{tabular}

esting to note that the univariate $\theta$ estimates correlate about equally with $\theta_{1}$ and $\theta_{2}$ for all levels of $\theta$ correlation and for each model. The correlations between $\theta_{1}$ and $\hat{\theta}$ and between $\theta_{2}$ and $\hat{\theta}$ range from $.59\left(\rho_{\theta, \theta_{1}}=0.0\right)$ to $.95\left(\rho_{0, \theta_{3}}=.9\right)$. The correlations between $\hat{\theta}$ and the average of $\theta_{1}, \theta_{2}$ are all quite high, ranging from .89 to .97 .

These results parallel the MADs: As the correlation between $\theta$ dimensions increases, the $\mathrm{MAD}$ values decrease. Thus as the data become more unidimensional, the MAD and correlational values support the conclusion that both programs appear to align the univariate scale about equidistant from the two $\theta$ axes.

For the compensatory datasets, correlations and MAD values for a (univariate discrimination) are displayed in Table 4. As the correlation between $\theta$ s increases, the correlation between $\hat{a}$ and $a_{1}$ and between $\hat{a}$ and $a_{2}$ approaches 0 for both LOGIST and BLOG. MAD values between the discrimination estimates and parameters were slightly higher for BILOG in all correlational conditions. For both programs, the correlation between $\hat{b}$ and $d$ was -.99 for all datasets. This suggests that the pattern of difficulty between the individual items is recoverable to a high degree.

Correlations and average MAD values for the discrimination and difficulty parameters and their es- timates for the noncompensatory datasets are in Table 5. The pattern of correlations between discrimination parameters and estimates is similar to that of the compensatory data. The correlations between $\hat{b}$ and $b_{1}$ are all .99, while the correlations between $\hat{b}$ and $b_{2}$ range from $r=.38$ to $r=.42$ for both LOGIST and BILOG. This suggests that a stronger relationship exists between Dimension 1 and the estimated unidimensional ability for the noncompensatory data. This may also be due to the restricted range of $b_{2}$ values.

In both the compensatory and noncompensatory datasets, the $\hat{a}$ s correlated positively with $a_{1}$ and negatively with $a_{2}$, except for the $p_{\theta_{1} \theta_{2}}=.9$ case. Noticeable differences exist between the MAD values for the noncompensatory discrimination parameters and estimates for BLOG and LOGIST. For LOGIST the average absolute differences of both $a_{1}-\hat{a}$ and $a_{2}-\hat{a}$ range from .80 to .86 , while the range is .32 to .38 for BILOG. For both calibration programs the correlations between $\hat{a}$ and $a_{2}$ are negative, except for the $\rho_{\theta_{1} \theta_{2}}=.9$ case in which the pattern reverses.

\section{Conclusions}

Differences between the item response surfaces for each model when the item parameters are matched 
Figure 6

Centroids for the LOGIST Calibrated Response Sets Among the Two-Dimensional Abilities

(a) Compensatory Response Sets

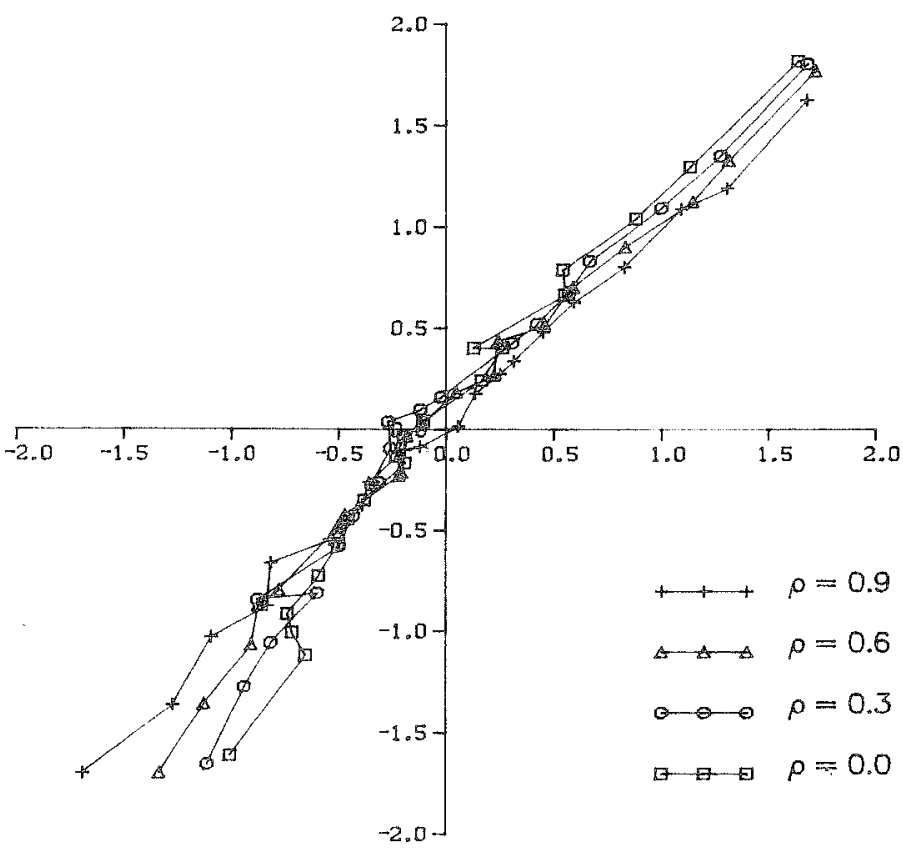

(b) Noncompensatory Response Sets

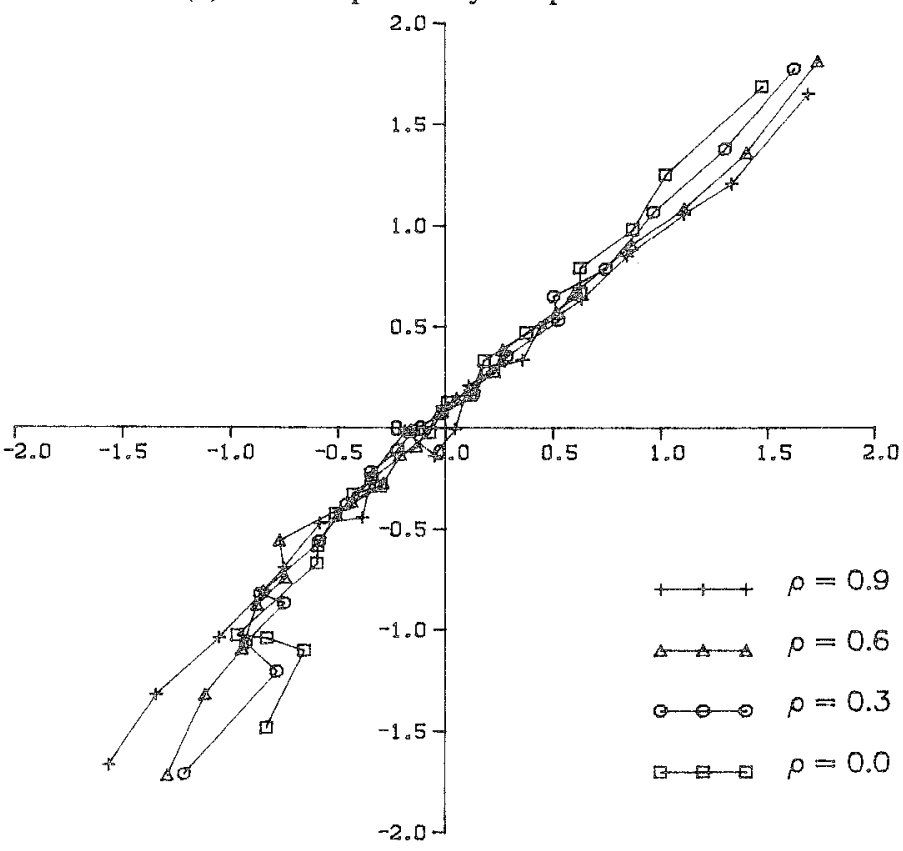

Downloaded from the Digital Conservancy at the University of Minnesota, http://purl.umn.edu/93227. requires payment of royalties through the Copyright Clearance Center, http://www.copyright.com/ 
Figure 7

Centroids for the BILOG Calibrated Response Sets Among the Two-Dimensional Abilities

(a) Compensatory Response Sets

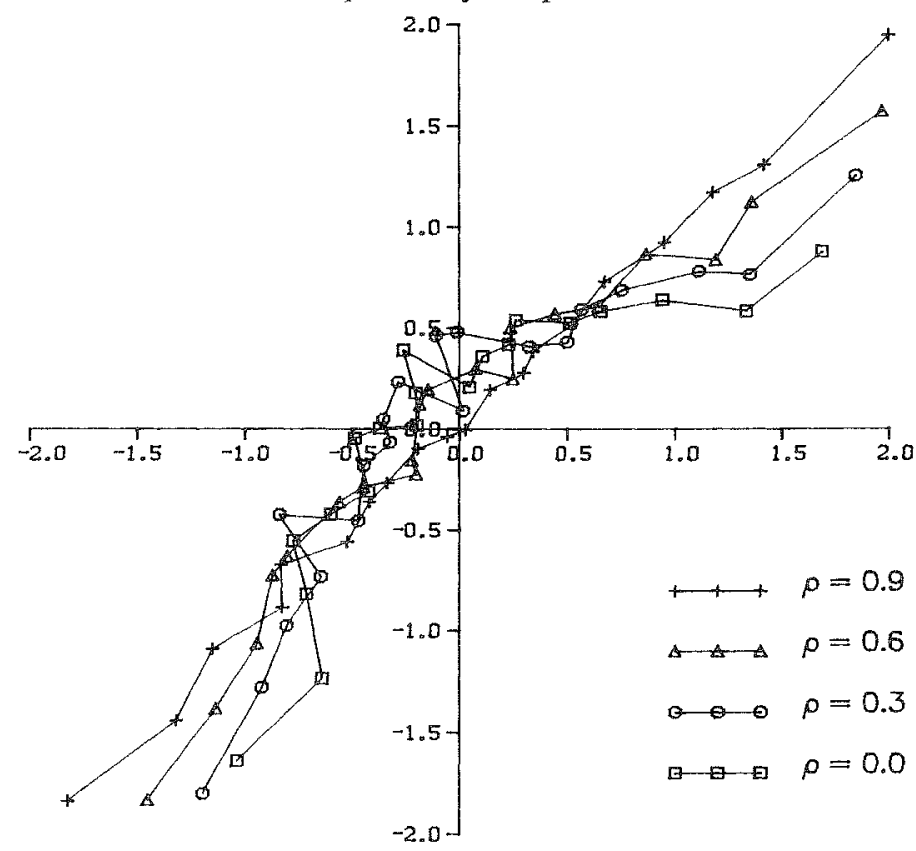

(b) Noncompensatory Response Sets

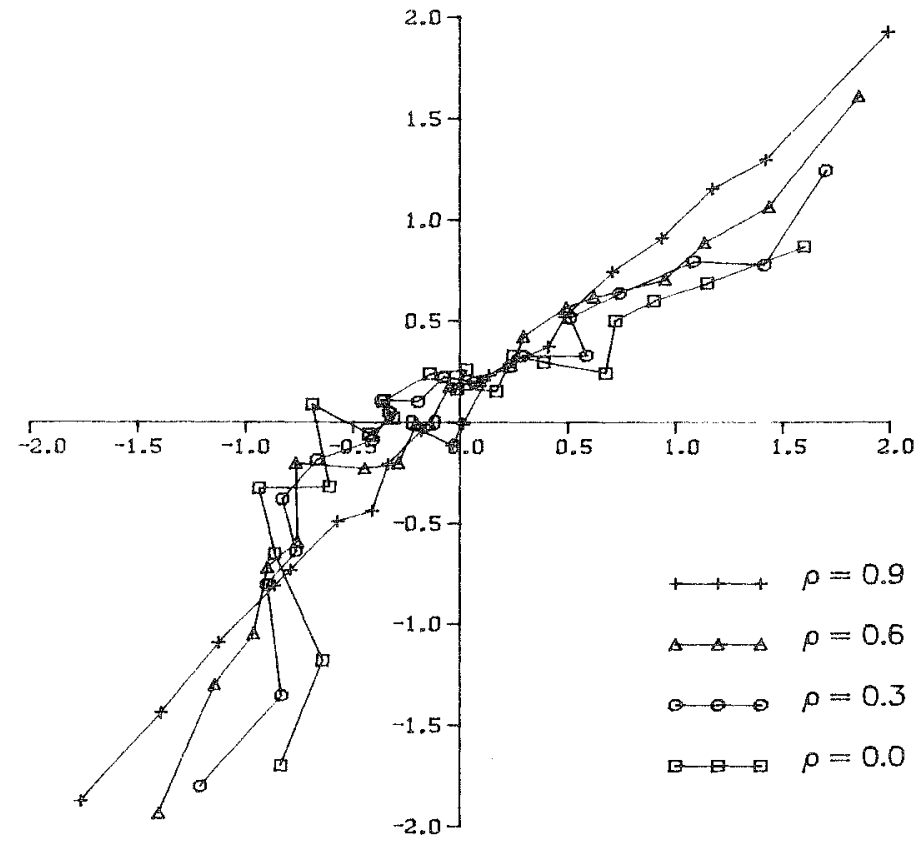

Downloaded from the Digital Conservancy at the University of Minnesota, http://purl.umn.edu/93227. requires payment of royalties through the Copyright Clearance Center, http://www.copyright.com/ 
Table 3

Correlations and Mean Absolute Differences Among $\hat{\theta}, \theta_{1}$ and $\theta_{2}$ By Levels of Correlation for Compensatory and Noncompensatory Darasets

\begin{tabular}{|c|c|c|c|c|c|c|}
\hline & & & & $\Sigma\left|\theta_{1}-\hat{\theta}\right|$ & $\Sigma\left|\theta_{2}-\hat{\theta}\right|$ & $\Sigma\left|\theta_{\text {avg }}-\hat{\theta}\right|$ \\
\hline$\rho\left(\theta_{1}, \theta_{2}\right)$ & $r\left(\hat{\theta}, \theta_{1}\right)$ & $r\left(\hat{\theta}, \theta_{2}\right)$ & $r\left(\hat{\theta}, \theta_{\mathrm{avg}}\right)$ & $k$ & $k$ & $k$ \\
\hline \multicolumn{7}{|c|}{ Compensatory Data: LOGIST } \\
\hline 0.0 & .67 & .64 & .94 & .65 & .67 & .34 \\
\hline .3 & .76 & .76 & .95 & .53 & .53 & .27 \\
\hline .6 & .85 & .85 & .96 & .42 & .42 & .23 \\
\hline .9 & .94 & .94 & .97 & .26 & .27 & .20 \\
\hline \multicolumn{7}{|c|}{ Compensatory Data: BILOG } \\
\hline 0.0 & .68 & .64 & .94 & .63 & .65 & .32 \\
\hline .3 & .78 & .76 & .95 & .53 & .54 & .28 \\
\hline .6 & .87 & .86 & .96 & .43 & .44 & .25 \\
\hline .9 & .95 & .95 & .97 & .28 & .28 & .21 \\
\hline \multicolumn{7}{|c|}{ Noncompensatory Data: LOGIST } \\
\hline 0.0 & .65 & .60 & .89 & .66 & .70 & .40 \\
\hline .3 & .76 & .72 & .92 & .54 & .58 & .32 \\
\hline .6 & .85 & .84 & .95 & .42 & .43 & .25 \\
\hline .9 & .94 & .94 & .96 & .27 & .28 & .21 \\
\hline \multicolumn{7}{|c|}{ Noncompensatory Data: BILOG } \\
\hline 0.0 & .67 & .59 & .90 & .62 & .67 & .35 \\
\hline .3 & .77 & .73 & .93 & .53 & .56 & .29 \\
\hline .6 & .86 & .85 & .95 & .42 & .44 & .26 \\
\hline .9 & .94 & .94 & .97 & .28 & .29 & .22 \\
\hline
\end{tabular}

appear to be minimal, and they exist at places on the $\theta_{1}, \theta_{2}$ plane where very few examinees would be expected to be found. Mean $p$ values for the eight sets were identical, and the matches on bi- serial correlations were almost identical for the $\rho_{\theta_{1} \theta_{2}}=.9$ case. Thus, the least-squares matching procedures appear to be an excellent method of matching the two multidimensional IRT models.

Table 4

Correlations and Mean Absolute Differences Between LOGIST and BILOG Estimates and Parameters Under the Compensatory Model

\begin{tabular}{|c|c|c|c|c|c|}
\hline & & & & $\Sigma\left|a_{1}-\hat{a}\right|$ & $\Sigma\left|a_{2}-\hat{a}\right|$ \\
\hline$\rho\left(\theta_{1}, \theta_{2}\right)$ & $r\left(\hat{a}, a_{1}\right)$ & $r\left(\hat{a}, a_{2}\right)$ & $r(\hat{B}, d)$ & $k$ & $k$ \\
\hline \multicolumn{6}{|l|}{ LOGIST } \\
\hline 0.0 & .30 & -.30 & -.99 & .40 & .45 \\
\hline .3 & .26 & -.26 & -.99 & .41 & .45 \\
\hline .6 & .17 & -.17 & -.99 & .41 & .44 \\
\hline .9 & -.07 & .07 & -.99 & .42 & .43 \\
\hline \multicolumn{6}{|l|}{ BILOG } \\
\hline 0.0 & .26 & -.26 & -.99 & .48 & .52 \\
\hline .3 & .18 & -.18 & -.99 & .49 & .50 \\
\hline .6 & .19 & -.19 & -.99 & .48 & .50 \\
\hline .9 & -.04 & .04 & -.99 & .48 & .48 \\
\hline
\end{tabular}


Table 5

Correlations and Mean Absolute Differences Between LOGIST and BILOG Estimates and Parameters Under the Noncompensatory Mode1

\begin{tabular}{|c|c|c|c|c|c|c|c|c|}
\hline & & & & & $\Sigma\left|a_{1}-\hat{a}\right|$ & $\Sigma\left|a_{2}-\hat{a}\right|$ & $\Sigma\left|b_{1}-\hat{b}\right|$ & $\Sigma \mid b_{2}-\hat{b}$ \\
\hline$\rho\left(\theta_{1}, \theta_{2}\right)$ & $r\left(\hat{a}, a_{1}\right)$ & $r\left(\hat{a}, a_{2}\right)$ & $r\left(\hat{b}, b_{1}\right)$ & $r\left(\hat{b}_{3} b_{2}\right)$ & $k$ & $k$ & $k$ & $k$ \\
\hline \multicolumn{9}{|l|}{ LOGIST } \\
\hline 0.0 & .31 & -.23 & .99 & .42 & .86 & .82 & .67 & .87 \\
\hline .3 & .27 & -.22 & .99 & .41 & .85 & .81 & .67 & .87 \\
\hline .6 & .19 & -.14 & .99 & .40 & .84 & .80 & .67 & .86 \\
\hline .9 & -.07 & .06 & .99 & .38 & .84 & .80 & .67 & .85 \\
\hline \multicolumn{9}{|l|}{ BILOG } \\
\hline 0.0 & .28 & -.21 & .99 & .42 & .35 & .38 & .67 & .86 \\
\hline .3 & .19 & -.17 & .99 & .41 & .33 & .37 & .67 & .86 \\
\hline .6 & .19 & -.20 & .99 & .40 & .32 & .35 & .67 & .86 \\
\hline .9 & -.04 & -.01 & .99 & .39 & .32 & .35 & .67 & .85 \\
\hline
\end{tabular}

However, confounding of difficulty with dimensionality, which was reported in Reckase et al. (1986), was replicated only for the BrLOG calibration of response data in which $\rho_{\theta_{1} \theta_{2}}$ was closer to 0 . The "wrap-around" effect of the $\theta_{1}, \theta_{2}$ centroids did not occur for any of the LOGIST estimation runs. However, in the Reckase et al. study the items only measured $\theta_{1}$ and $\theta_{2}$, whereas in this study each item measured a combination of $\theta_{1}$ and $\theta_{2}$ to varying degrees. Thus the confounding was not as great as in the Reckase et al. study. Another possible explanation may be the method of estimation. Perhaps the marginal maximum likelihood (MML) procedure of BILOG is more sensitive to the confounding of difficulty and dimensionality.

Confounding of difficulty appeared to have the same effect on $\theta$ estimates for both the compensatory and noncompensatory datasets. Despite different item information patterns, as seen in the INFLINE plots, the orientation of the centroids appeared to be the same for both calibration programs.

The correlations among $\theta$ parameters and estimates suggest that as the relationship between the two ability dimensions becomes more linear, the data become unidimensional in a sense. As $p_{\theta_{1} \theta_{2}}$ approached $.9, \hat{\theta}$ correlated in the mid-.90s with $\theta_{1}$ and $\theta_{2}$. This was confirmed by the plots of the $\theta_{1}, \theta_{2}$ centroids and the correlations of the discriminating parameters with their estimates. The correlations between $\hat{a}$ and $a_{1}$ and between $\hat{a}$ and $a_{2}$ became closer as the correlation between $\theta_{1}$ and $\theta_{2}$ increased. Similarly, as $p_{\theta_{1} \theta_{2}}$ approached .9, the centroids appeared to align themselves along a $45^{\circ}$ line. Both of these results suggest that $\theta_{1}$ and $\theta_{2}$ were being measured equally.

These results differ slightly from those of Way et al. (1988) and Ansley and Forsyth (1985), in which the relationship between $\hat{\theta}$ and $\theta_{1}$ was found to be stronger than the relationship between $\hat{\theta}$ and $\theta_{2}$ for low levels of $\rho_{\theta_{1} \theta_{2}}$. However, the relationship Way et al. and Ansley and Forsyth found between the estimated $\theta$ values and the average of the true $\theta_{1}, \theta_{2}$ values was found to be even stronger in this study. Both of these findings may be a function of the method used to generate item parameters in these prior studies, which differed from the method used in this study.

The plots of the $\theta_{1}, \theta_{2}$ centroids for the $20 \hat{\theta}$ quantiles revealed differences between the two estimation programs. The centroid plot for LOGIST revealed only a slight confounding effect as $p_{\theta_{1} \theta_{2}}$ became closer to 0 . However, $\theta_{1}, \theta_{2}$ centroids for BILOG's $\hat{\theta}$ display a much sharper wrapping around about the negative $\theta_{2}$ axis and the positive $\theta_{1}$ axis, especially when $\rho_{\theta_{1} \theta_{2}}=0.0$. Thus, BILOG appears 
to be more sensitive to the confounding of difficulty with dimensionality for both multidimensional models.

Several directions for future research are suggested by this study. One area would be to systematically vary test information with different twodimensional $\theta$ distributions to determine how the interaction affects the orientation of the univariate $\theta$ scale in the two-dimensional plane. Also, differences between maximum likelihood and MML estimation of multidimensional response data need to be further explored. Perhaps for the datasets used in this study, BILOG mapped different $\theta_{1} \theta_{2}$ combinations than LOGIST onto the univariate $\theta$ scale because the BILOG combinations accounted for more variation in the item responses from examinees in that neighborhood.

\section{Refererences}

Ansley, T. N., \& Forsyth, R. A. (1985). An examination of the characteristics of unidimensional IRT parameter estimates derived from two-dimensional data. Applied Psychological Measurement, 9, 37-48.

Carlson, J. E. (1987). Multidimensional item response theory estimation: A computer program (Research $\mathbb{R e}-$ port 87-19). Iowa City IA: American College Testing Program.

Hambleton, R. K., \& Murray, L. N. (1983). Some goodness of fit investigations for item response models. In R. K. Hambleton (Ed.), Applications of item response theory. Vancouver $\mathrm{BC}$ : Educational Research Institute of British Columbia.

McKinley, R. L., \& Reckase, M. D. (1983). MAXLOG: A computer program for the estimation of the parameters of a multidimensional logistic model. Behavior Research Methods and Instrumentation, 15, 389-390.

Mislevy, R. J. \& Bock, R. D. (1982). BLLOG, maximum likelihood item analysis and test scoring: Logistic model. Mooresville IN: Scientific Software, Inc.

Mislevy, R. J., \& Stocking, M.L. (1989). A consumer's guide to LOGIST and BILOG. Applied Psychological Measurement, 13, 57-75.
Reckase, M. D. (1985). The difficulty of test items that measure more than one ability. Applied Psychological Measurement, 9, 401-412.

Reckase, M. D., Carlson, J. E., Ackerman, T. A., \& Spray, J. A. (1986, June). The interpretation of unidimensional IRT parameters when estimated from multidimensional data. Paper presented at the annual meeting of the Psychometric Society, Toronto.

Sympson, J. B. (1978). A model for testing with multidimensional items. In D. J. Weiss (Ed.), Proceedings of the 1977 Computerized Adaptive Testing Conference (pp. 82-98). Minneapolis: University of Minnesota, Department of Psychology, Psychometric Methods Program.

Traub, R. E. (1983). A priori consideration in choosing an item response model. In R. K. Hambleton (Ed.), Applications of item response theory (pp. 57-70). Vancouver BC: Educational Research Institute of British Columbia.

Way, W. D., Ansley, T. N., \& Forsyth, R. A. (1988). The comparative effects of compensatory and noncompensatory two-dimensional data on unidimensional IRT estimates. Applied Psychological Measurement, 12, 239-252.

Wingersky, M. S., Barton, M. A., \& Lord, F. M. (1982). LOGIST user's guide. Princeton NI: Educational Testing Service.

Yen, W. M. (1984). Effects of local item dependence on the fit and equating performance of the three-parameter logistic model. Applied Psychological Measurement, $8,125-145$.

\section{Aching}

The author thanks Judy Spray, Mark Reckase, Jim Carlson, and Brad Sympson for their useful comments.

\section{Author's Address}

Send requests for reprints or further information to Terry A. Ackerman, ACT, P.O. Box 168, lowa City IA 52243, U.S.A. 